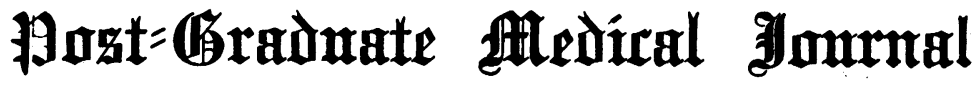

\author{
$\longrightarrow$ \\ LONDON： FEBRUARY I, I940. \\ FOREWORD \\ to the Symposium on Focal Sepsis
}

By The Rt. Hon. LORD HORDER, G.C.V.O.

I have been asked to write a short Foreword to introduce a series of Essays from a number of colleagues upon this subject, and I do so with pleasure. I have grown up, as it were, pari passu with the development of the doctrine of focal sepsis and have watched its growth with more than mere academic interest.

If I were to attempt a definition of the subject it would be this. "Focal sepsis is a term used to signify the presence of a local and chronic infection, itself yielding few or no symptoms, yet resulting in a state of toxæmia, with or without mild bacteriæmia, and leading to a number of general or remote local disturbances."

The microbe most commonly responsible for the condition is the streptococcus and most often of the non-hæmolytic kind. Coliform bacilli probably come next in frequency on the list, then the gonococcus; Pfeiffer's bacillus is concerned much less frequently. The process is not characteristic of the staphylococcus.

In the majority of the cases the focus is " closed " and not "open," though this distinction is not to be regarded as a rigid one. The most common sites are connected with the teeth, the nasal sinuses and the tonsils. Pyorrhœa alveolaris (suppurative parodontitis) is an open focus; apical sepsis, which may be paradoxically called " pyorrhœa sicca," is a closed one. The tonsils, like the teeth, present both open and closed examples of infection. In the alimentary tract the common sites are the gall-bladder and the appendix, but colon diverticuli may also provide the focus. Some authorities include diffuse sub-infection of the bowel in this category. In the genito-urinary tract the organs most concerned are the prostate, the fallopian tubes and the renal pelvis. In bronchiectasis a similar state of affairs exists in the respiratory system.

Since the strains of micro-organisms involved are nearly always of the nonvirulent kind suppuration is by no means always present in the primary focus. The process is generally to be regarded as a "sub-infection" (Adami). It is quite impossible to say in how many of the cases the microbe is present in the secondary lesions, but it is probably a true view to take that in this series of cases there exists every transition from a simple toxæmia to a low-grade pyæmia. In the great majority of cases blood-cultures are sterile, but this does not, of course, exclude the occasional passage of the causative microbe into the circulation. 
The effects are widespread. In some cases it is a state of general ill-health which attracts attention: fatigue, headache, backache, insomnia and neurasthenia. In others the symptoms may be referred to special organs or tissues. Of these fibrositis is the most common: arthritis, peri-neuritis, myalgia, and "chronic rheumatism" in all its forms. Disorders of the heartbeat are common. The skin may be affected in a number of ways: acneform and rosaceous eruptions, erythemata and pruritus without objective lesions. Iritis, often recurrent in type, is most often due to focal sepsis, whether gonococcal or streptococcal.

Although the process is, in the main, a chronic one, it may arise with acute symptoms or, more characteristically, may show acute exacerbations. Thus, a knee may flare rather rapidly, with the development of an acute synovitis or arthritis. Iritis is, more often than not, acute.

The blood may show little, or no, change. But if there is an acute secondary lesion such as arthritis, a moderate leucocytosis is usually present; and in some of the cases presenting constitutional changes there is a mild, but definite, degree of anæmia and a change in the differential white count which is rather characteristic - an absolute rise in the hyaline cells so that the percentages of polymorphonuclear and hyaline cells tend to become equal or even to shew slight inversion of the normal proportions. Although pyrexia is not often a feature, the number of cases in which a mild degree of it is present is found to be larger than is realised when a proper record is taken.

Experience alone teaches in what type of patient the answer to the problem presented lies in the uncovering of a state of focal sepsis. The nature of the process should be constantly in the practitioner's mind, and a systematic search should be made, having regard to the various sites just enumerated.

The above is a brief outline of the subject (omitting reference to diagnosis and treatment) as generally accepted. But the concept may be enlarged and still be found to conform to facts; thus, infected new growths and sepsis in association with pulmonary tuberculosis behave in similar fashion to the more commonly described foci, and subacute bacterial endocarditis may be regarded as a form of focal sepsis in an inaccessible situation.

I think Dr. Joekes is correct in saying that the first discovery which drew our attention to the subject of focal sepsis, in this country at all events, was the clinical experience associating the cure of certain cases of "rheumatism" with the extraction of septic teeth. Similar experiences soon attracted notice in relation to other organs and tissues, until it became clear that we were faced with a principle in the elucidation of which clinician and pathologist joined hands. As Mr. Bourne says, "the new idea was hard worked in the beginning "; but that is the fate of all new ideas. Doubtless there are some who still work it too hard, neglecting to give due consideration to nervous, metabolic, hormonal, environmental and heredity factors, but in the practice of most of us the pendulum has come to rest at a point of equilibrium.

A study of these papers must surely convince any who are still sceptical that a consideration of the subject to-day constitutes an important part of the routine examination and treatment of many patients who come to us, and the best proof of the validity of the principle is the fact that its pursuit in actual practice is 
justified again and again by the cure or relief of the troubles from which these patients suffer.

Some of these essays may seem to the reader to be somewhat prolix, but it is clear that the Editor has decided to allow each expert to " let himself go " to the full extent of his own experience and ideas. I think this is wise. The result is that we have, I believe, in this Symposium the most complete and authoritative account of the matter yet available. The Post-Graduate Journal has performed a similar service to medicine more than once before and the Editor is to be congratulated upon the achievement of one more signal success.

\section{Post-Graduate News.}

The following Courses have been arranged:-

For M.R.C.P. Candidates: (I) Chest Diseases at the Brompton Hospital on Mondays and Thursdays at 5.0 p.m., January 29th to February 22nd, I940, by Dr. F. P. Lee Lander. The course consists of clinical cases and X-rays, and is limited to six post-graduates. Fee, to Members of the Fellowship of Medicine; $£ 3$ I3s. 6d., and to non-Members, $£ 44 \mathrm{~s}$. od. A similar course will be given by Dr: J. G. Scadding, from February 26th to March 2Ist. (2) Neurology, at the West End Hospital for Nervous Diseases, I.P. Department, Gloucester Gate, Regents Park, every afternoon at 2.0 p.m., February $5^{\text {th }}$ to I6th, I940. The course consists of a clinical demonstration, followed by a lecture, each afternoon. Fee, to Members of the Fellowship of Medicine, $£ 33$ s. od., and to non-Members, $£ 3$ I3s. 6d.

For F.R.C.S. Candidates: (I) Comprehensive Course, at the Royal Cancer Hospital, every morning 9.30 to I.o, March 4th to April I9th, by Mr. Ronald Raven. The course includes clinical demonstrations, tutorials, museum and X-ray demonstrations, etc. Fee, to Members of the Fellowship of Medicine, $£ 77 \mathrm{~s}$. od., and to non-Members, $£ 7$ I7s. 6d. (2) Operative Surgery, at the Royal Cancer Hospital, in the afternoons (or by arrangement) April 8th to May 3rd, by Mr. Ronald Raven. Fee, to Members of the Fellowship of Medicine, £Io ros. od., and to non-Members, £I2 I2s. od.

Other courses will be advertised as and when arranged. 